\section{CUIDADORES INFORMALES DE PACIENTES CON ENFERMEDADES CEREBROVASCULARES}

\section{INFORMAL CAREGIVERS OF PATIENTS WITH CEREBROVASCULAR DISEASES}

\author{
Giancarlo A. Valle ${ }^{1, a}$, Julio A. Poterico $2, a$, \\ Renato Quispe ${ }^{2, a}$
}

Sr. Editor. La incidencia de enfermedades cerebrovasculares (ECV) se ha incrementado en las últimas décadas. Mundialmente, 5,5 millones de muertes al año se deben a esta enfermedad, con 44 millones de años de vida perdidos por discapacidad (DALYs). En la última década, los países de medianos y bajos ingresos alcanzaron el mayor número de DALYs y la mayor prevalencia de ECV, lo cual se espera que aumente en América Latina y Asia ${ }^{(1)}$.

Las ECV pueden causar en quienes la padecen discapacidad severa y dependencia para el desarrollo de las actividades de la vida diaria. Esto representa un cambio repentino y significativo en la vida de sus familiares, quienes asumen la mayor parte de la responsabilidad y cuidado del paciente ${ }^{(2)}$. El shock variará según la evolución clínica del paciente y la repercusión emocional en los familiares, los llamados cuidadores informales.

Los cuidadores informales necesitan aprender a afrontar las discapacidades de sus familiares con ECV. En situaciones de mayor grado de dependencia post ECV, los cuidadores pueden invertir gran parte de tiempo en la atención del paciente, llegando, en muchos casos, a perder sus empleos ${ }^{(2)}$. Durante esta adaptación podrían también renunciar a sus actividades recreacionales, impactando en sus relaciones sociales y afectando su autonomía, satisfacción e, incluso, llegando al agotamiento emocional (3). Así, los cuidadores informales, en su mayoría con poca o nula capacitación, estarían en mayor riesgo de sufrir algún trastorno mental; exacerbando, incluso, condiciones físicas preexistentes ${ }^{(3)}$.

1 Facultad de Medicina, Universidad Peruana Cayetano Heredia. Lima, Perú.

2 CRONICAS Centro de Excelencia en Enfermedades Crónicas, Universidad Peruana Cayetano Heredia. Lima, Perú.

a Médico cirujano

Recibido: 16-12-13 Aprobado: 18-12-13

Citar como: Valle GA, Poterico JA, Quispe R. Cuidadores informales de pacientes con enfermedades cerebrovasculares [carta]. Rev Peru Med Exp Salud Publica. 2014;31(1):169-70.
Intervenciones en países desarrollados han tratado de mejorar la calidad de vida de los cuidadores informales; brindándoles consejería, soporte psicológico, social y tecnológico para mejorar sus actividades cotidianas de atención ${ }^{(4)}$. Los estudios concernientes a este tema sugieren resultados positivos en los familiares de pacientes con ECV, tales como disminución de sintomatologías depresivas, aumento del conocimiento sobre ECV, incremento de la satisfacción al realizar actividades de cuidado, mejora de las habilidades para resolver problemas, mejora en el funcionamiento familiar, actividades sociales y calidad de vida del cuidador (4). Sin embargo, la heterogeneidad de los estudios hace difícil la comparación de los mismos para dar conclusiones ${ }^{(4)}$.

En Latinoamérica se estima que más del $50 \%$ de los cuidadores informales dejan sus trabajos por lo que luego enfrentan problemas financieros ${ }^{(5)}$. Surgen preguntas debido a falta de evidencia científica en Latinoamérica acerca de cómo están siendo afectados y protegidos los cuidadores de pacientes con ECV: ¿están los cuidadores informales incluidos en las políticas públicas?; y, ¿quién cuida de ellos?

Con esta problemática desatendida, exhortamos a las instituciones de salud latinoamericanas a enfatizar la vigilancia y manejo de los cuidadores informales de pacientes con secuela de ECV, facilitando programas de consejería e incluyéndolos en las guías de manejo de pacientes con ECV con adaptación cultural y contextual. El hecho de que un familiar cuide a un ser querido no debería acarrear padecimiento adicional, puesto que esto podría afectar la estructura familiar, social y económica de nuestras sociedades.

Fuentes de financiamiento: autofinanciado.

Conflictos de interés: los autores declaran no tener conflictos de interés.

\section{REFERENCIAS BIBLIOGRÁFICAS}

1. Mukherjee D, Patil CG. Epidemiology and the global burden of stroke. World Neurosurg. 2011;76(6 Suppl):S85-90. doi: 10.1016/j.wneu.2011.07.023.

2. Ferri CP, Schoenborn C, Kalra L, Acosta D, Guerra M, Huang $\mathrm{Y}$ et al. Prevalence of stroke and related burden among older people living in Latin America, India and China. J Neurol Neurosurg Psychiatry. 2011;82(10):1074-82. doi: 10.1136/ jnnp.2010.234153.

3. Anderson CS, Linto J, Stewart-Wynne EG. A populationbased assessment of the impact and burden of caregiving for long-term stroke survivors. Stroke. 1995;26(5):843-9. 
4. Visser-Meily A, van Heugten C, Post M, Schepers V, Lindeman E. Intervention studies for caregivers of stroke survivors: a critical review. Patient Educ Couns. 2005;56(3):257-67.

5. Morais HC, Soares AM, Oliveira AR, Carvalho CM, da Silva MJ, de Araujo TL. Burden and modifications in life from the perspective of caregivers for patients after stroke. Rev Lat Am Enfermagem. 2012;20(5):944-53.

Correspondencia: Renato Quispe Ayala

Dirección: Calle Jorge Muelle 433 Dpto. 504, San Borja, Lima 41, Perú.

Teléfono: 987760463

Correo electrónico: renato.quispe@upch.pe

\section{GENERANDO INFORMACIÓN: ¿SABEMOS CUÁNTO ES EL CONSUMO PROMEDIO DE SAL Y CUÁLES SON SUS FUENTES?}

\author{
GENERATING INFORMATION: WHAT IS THE \\ AVERAGE CONSUMPTION OF SALT AND \\ WHAT ARE THE SOURCES?
}

\author{
Lorena Saavedra-Garcia1,a, \\ Antonio Bernabé-Ortiz ${ }^{1,2, b}$, \\ Francisco Diez-Canseco ${ }^{1, c}$, J. Jaime Miranda ${ }^{1,3, d}$
}

Sr. Editor. El mundo vive una transición alimentarianutricional caracterizada por el incremento del consumo de azúcares simples, grasas saturadas y sodio (el cual tiene como fuente principal la sal). Esta situación ha impactado negativamente en la salud, incrementando la prevalencia de enfermedades crónicas. Ante ello, algunos gobiernos han implementado políticas públicas para reducir el consumo de dichos compuestos ${ }^{(1)}$.

Para poder establecer indicadores de éxito y evaluar los progresos de estas iniciativas, es necesario contar

\footnotetext{
CRONICAS Centro de Excelencia en Enfermedades Crónicas, Universidad Peruana Cayetano Heredia. Lima, Perú.

2 Unidad de Epidemiología, Facultad de Salud Pública y Administración, Universidad Peruana Cayetano Heredia. Lima, Perú.

3 Departamento de Medicina, Facultad de Medicina, Universidad Peruana Cayetano Heredia. Lima, Perú.

a Licenciada en Nutrición, ${ }^{\mathrm{b}}$ médico magíster en Salud Pública, ${ }^{\mathrm{c}}$ psicólogo magíster en Salud Pública, ' médico magíster y doctor en Epidemiología

Recibido: 16-12-13 Aprobado: 18-12-13
}

Citar como: Saavedra-García L, Bernabé-Ortiz A, Diez-Canseco F, Miranda JJ. Generando información: ¿sabemos cuánto es el consumo promedio de sal y cuáles son sus fuentes? [carta]. Rev Peru Med Exp Salud Publica. 2014;31(1):170-1. con información adecuada. Sobre el consumo de sodio, por ejemplo, es necesario conocer cuál es su consumo diario promedio, cuáles son las principales fuentes alimentarias que contribuyen con sodio en la dieta (tanto naturales como procesadas), y cuáles son las características a nivel poblacional que determinan estos hábitos de consumo. A nivel mundial, el consumo diario de sal varía de 9 a $12 \mathrm{~g}^{(1)}$, cerca del doble de los $5 \mathrm{~g} /$ día recomendados por la Organización Mundial de la Salud (OMS) ${ }^{(2)}$. Se sabe que la principal fuente de sodio en los países desarrollados son los alimentos industrializados, que aportan alrededor del $75 \%$ del consumo total de este elemento ${ }^{(3)}$. Países como Estados Unidos, por ejemplo, cuentan con reportes sobre el consumo promedio de sal de su población ${ }^{(3)}$ esta información, sumada a su compromiso con mejorar la salud de sus habitantes, lo que les ha permitido plantear acciones concretas frente a este problema ${ }^{(1)}$. Desde la década pasada, se han creado iniciativas para disminuir el consumo de sodio. Finlandia y Reino Unido, por ejemplo, han realizado acuerdos con la industria para reducir el contenido de dicho elemento de forma paulatina en los alimentos procesados (1). Recientemente, la OMS ha buscado establecer indicadores de reducción del consumo de sal, definiendo metas concretas, como por ejemplo una reducción del $30 \%$ para el $2025^{(4)}$.

Sin embargo, los datos sobre la ingesta de sodio no están disponibles en muchos países en desarrollo, donde además el modelo alimentario y los patrones de consumo no son iguales a los del resto del mundo. En Brasil se reportó, para el periodo 2002-2003, que 71,5\% de la ingesta de sodio provenía de la sal agregada durante la cocción de las comidas ${ }^{(5)}$, datos opuestos a los de países desarrollados. En Perú no hay datos actualizados sobre la ingesta de sal o sus fuentes; es decir, no sería posible monitorear nuestro avance mediante los indicadores de reducción del consumo de sodio planteadas en acuerdos globales.

Perú es un caso particular pues en él coexisten distintos patrones alimentarios, que cambian de región en región, lo que supone contar con una amplia lista de alimentos que aportan sal a la dieta. Es insoslayable que la sal es parte de nuestra cultura y es un potenciador del sabor muy utilizado. Dada la importancia de la sal en la dieta, consideramos relevante estudiar, además, las características específicas de la población con respecto a la palatabilidad, obstáculo a vencer para conseguir una reducción del consumo de sal.

Se hace indispensable desarrollar acciones para poder adoptar y monitorear, con la rigurosidad necesaria, aquellas iniciativas que busquen reducir el consumo de sal y poder participar de los procesos de monitorización, 